\title{
Homotopy type of the neighborhood complexes of graphs of maximal degree at most 3 and 4-regular circulant graphs
}

\author{
Samir Shukla* \\ Department of Mathematics \\ Indian Institute of Technology Bombay \\ Mumbai, India \\ samirs@math.iitb.ac.in, samirshukla43@gmail.com
}

Submitted: Dec 18, 2017; Accepted: Mar 19, 2019; Published: Apr 5, 2019

(C) The author. Released under the CC BY-ND license (International 4.0).

\begin{abstract}
To estimate the lower bound for the chromatic number of a graph $G$, Lovász associated a simplicial complex $\mathcal{N}(G)$ called the neighborhood complex and related the topological connectivity of $\mathcal{N}(G)$ to the chromatic number of $G$. More generally he proved that the chromatic number of $G$ is bounded below by the topological connectivity of $\mathcal{N}(G)$ plus 3 .

In this article, we consider the graphs of maximal degree at most 3 and 4-regular circulant graphs. We show that each connected component of the neighborhood complexes of these graphs is homotopy equivalent either to a point, to a wedge sum of circles, to a wedge sum of 2-spheres $S^{2}$, to $S^{3}$, to a garland of 2 -spheres $S^{2}$ or to a connected sum of tori.
\end{abstract}

Mathematics Subject Classifications: 05C15, 57M15

\section{Introduction}

The neighborhood complex, $\mathcal{N}(G)$ of a graph $G$ is the simplicial complex whose vertices are all non isolated vertices of $G$ and simplices are those subsets of $V(G)$, which have a common neighbor. The concept of neighborhood complex was introduced by Lovász [8] in his proof of the Kneser conjecture, that if we split the $n$-subsets of a $(2 n+k)$-elements set into $k+1$ classes, then one of the classes will contain two disjoint subsets. To prove this conjecture, Lovász first converted this set theoretic problem into an equivalent problem of

*The author was financially supported by the Indian Statistical Institute Bangalore, India, where this work was done. 
the computation of chromatic number of a class of graphs, called Kneser graphs and then related the connectivity of neighborhood complex to the chromatic number of graph.

A topological space $X$ is said to be $k$-connected if every map from a $m$-dimensional sphere $S^{m} \rightarrow X$ can be extended to a map from the $(m+1)$-dimensional ball $\mathbb{B}^{m+1} \rightarrow X$ form $m=0,1, \ldots, k$.

Theorem 1. (Lovász) If $\mathcal{N}(G)$ is $k$-connected, then $\chi(G) \geqslant k+3$.

Lovász generalized the notion of neighborhood complex to a polyhedral complex $\operatorname{Hom}(G, H)$, called the hom complex, for graphs $G$ and $H$. In particular $\operatorname{Hom}\left(K_{2}, G\right)$ and $\mathcal{N}(G)$ are homotopy equivalent. The 0-dimensional cells of $\operatorname{Hom}(G, H)$ are the graph homomorphisms from $G$ to $H$. For more details about hom complexes we refer the reader to [1]. In [3], Björner and Longueville showed that the neighborhood complexes of a family of vertex critical subgraphs of Kneser graphs, the stable Kneser graphs, are spheres up to homotopy. In [10], Nilakantan and author studied the neighborhood complexes of the exponential graphs $K_{n+1}^{K_{n}}$. In this article we compute the homotopy type of the neighborhood complexes of 4-regular circulant graphs.

Let $n \geqslant 2$ be a positive integer and $S \subset\{1,2, \ldots, n-1\}$. The circulant graph $C_{n}(S)$ is the graph, whose set of vertices $V\left(C_{n}(S)\right)=\{1,2, \ldots, n\}$ and any two vertices $x$ and $y$ adjacent if and only if $x-y(\bmod \mathrm{n}) \in S \cup-S$, where $-S=\{n-a \mid a \in S\}$. Circulant graphs are also Cayley graphs of $\mathbb{Z}_{n}$, the cyclic group on $n$ elements. Since $n \notin S, C_{n}(S)$ is a simple graph, i.e., does not contains any loop. Further, $C_{n}(S)$ are $|S \cup-S|$-regular graphs, here $|\cdot|$ denoting the cardinality. It can be easily verify that $C_{n}(S)$ is connected if and only if $S \cup-S$ generates $\mathbb{Z}_{n}$. In this article we restrict ourselves to $|S|=2$ and for the convenience of notation, we write $C_{n}(s, t)$ in place of $C_{n}(\{s, t\})$. Since $C_{n}(s, t)=C_{n}(n-s, t)=C_{n}(s, n-t)$, we assume that $s, t \leqslant \frac{n}{2}$.

\section{Statement of results}

We use the following definition of garland of topological spaces in Theorem 5 and Theorem 6. Let $X_{1}, X_{2}, \ldots, X_{n}$ be topological spaces. A topological space $X=\bigcup_{i=1}^{n} X_{i}$ is said to be a garland of $X_{1}, \ldots, X_{n}$, if $\left|X_{i} \cap X_{j}\right|=1$ when $|i-j|=1(\bmod n)$ and $X_{i} \cap X_{j}=\emptyset$ when $|i-j| \neq 1(\bmod n)$ for all $1 \leqslant i \neq j \leqslant n$.

Theorem 2. Let $G$ be a connected graph with maximal degree at most 3 and $G \neq K_{4}, T$ (see Figure 1a and 1b). Each connected component on $\mathcal{N}(G)$ is either contractible or homotopy equivalent to wedge sum of circles.

Let $n \geqslant 5$ and $s, t \in\left\{1,2, \ldots,\left\lfloor\frac{n}{2}\right\rfloor\right\}, s \neq t$. To compute the homotopy type of the neighborhood complexes of 4-regular circulant graphs we divide the set $I=\{\{s, t\} \mid s, t \in$ $\left.\left\{1,2, \ldots,\left\lfloor\frac{n}{2}\right\rfloor\right\}, s \neq t\right\}$ in to the following disjoint classes: $I_{1}=\{\{s, t\} \in I \mid n \in$ $\{2 s, 2 t, 2(s+t)\}\}, I_{2}=\left\{\{s, t\} \in I \backslash I_{1} \mid 3 s=t\right.$ or $\left.3 t=s\right\}, I_{3}=\left\{\{s, t\} \in I \backslash\left(I_{1} \cup I_{2}\right) \mid 3 s=\right.$ $5 t$ or $3 t=5 s\}$ and $I_{4}=I \backslash\left(I_{1} \cup I_{2} \cup I_{3}\right)$. 
Theorem 3. Let $\{s, t\} \in I_{1}$. Each connected component of $\mathcal{N}\left(C_{n}(s, t)\right)$ is homotopy equivalent to

(A) a point or wedge sum of circles, if $2 s=n$ or $2 t=n$.

(B) a point or $S^{1}$, if $2 s, 2 t \neq n$ and $2(s+t)=n$.

Theorem 4. Let $\{s, t\} \in I_{2}$. Each connected component of $\mathcal{N}\left(C_{n}(s, t)\right)$ is homotopy equivalent to

(A) $S^{3}$, if $3 s=t$ and $n=10 s($ or $3 t=s$ and $n=10 t)$.

(B) $S^{2} \vee S^{2}$, if $3 s=t$ and $n=12 s($ or $3 t=s$ and $n=12 t)$.

(C) wedge sum of circles, if $3 s=t$ and $n \neq 8 s, 10 s, 12 s$ (or $3 t=s$ and $n \neq 8 t, 10 t, 12 t)$.

Theorem 5. Let $\{s, t\} \in I_{3}$. Each connected component of $\mathcal{N}\left(C_{n}(s, t)\right)$ is homotopy equivalent to

(A) a garland of the 2 -dimensional spheres $S^{2}$, if $5 s=3 t$ and $n=4 t$ (or $5 t=3 s$ and $n=$ $4 s)$.

(B) $S^{2} \vee S^{2}$, if $5 s=3 t$ and $n=4 s($ or $5 t=3 s$ and $n=4 t)$.

(C) wedge sum of circles, if $5 s=3 t$ and one of $6 s$ or $\frac{14 s}{3}$ is equal to $n$ (or $5 t=3 s$ and one of 6 t or $\frac{14 t}{3}$ is equal to $n$ ).

(D) connected sum of tori, if $5 s=3 t$ and $4 s, 6 s, \frac{14 s}{3}, 4 t \neq n$ (or $5 t=3 s$ and $4 t, 6 t, \frac{14 t}{3}$, $4 s \neq n)$.

Theorem 6. Let $\{s, t\} \in I_{4}$. Each connected component of $\mathcal{N}\left(C_{n}(s, t)\right)$ is homotopy equivalent to

(A) either $S^{1}$ or $S^{3}$, if one of the $3 s-t, 3 t-s, 3 s+t$ or $3 t+s$ is equal to $n$.

(B) a garland of the 2-dimensional spheres $S^{2}$, if $3 s-t, 3 t-s, 3 s+t, 3 t+s \neq n$ and, $4 t=n$ or $4 s=n$.

(C) connected sum of tori, if $3 s-t, 3 t-s, 3 s+t, 3 t+s, 4 s, 4 t \neq n$.

The following theorem can be considered as a special case of Theorem 6 (C).

Theorem 7. Let $n=p q$, where $\operatorname{gcd}(p, q)=1$. Let $s, t \in\{1, \ldots, n-1\}$ such that $2 s, 2 t, 2(s+t), 3 s-t, 3 t-s, 3 s+t, 3 t+s, 4 s, 4 t \not \equiv 0(\bmod n)$. If $s=\frac{p-q}{2}$ and $t=\frac{p+q}{2}$ or, $s=\frac{p^{2}-q}{2}$ and $t=\frac{p^{2}+q}{2}$, then $\mathcal{N}\left(C_{n}(s, t)\right)$ is homotopy equivalent to torus. 


\section{Preliminaries}

\subsection{Graph}

A graph $G$ is a pair $(V(G), E(G))$, where $V(G)$ is the set of vertices of $G$ and $E(G) \subset$ $V(G) \times V(G)$ denotes the set of edges. If $(x, y) \in E(G)$, it is also denoted by $x \sim y$. A subgraph $H$ of $G$ is a graph with $V(H) \subset V(G)$ and $E(H) \subset E(G)$. For a subset $U \subset V(G)$, the induced subgraph $G[U]$ is the subgraph whose set of vertices $V(G[U])=U$ and the set of edges $E(G[U])=\{(a, b) \in E(G) \mid a, b \in U\}$.

A graph homomorphism from $G$ to $H$ is a function $\phi: V(G) \rightarrow V(H)$ such that, $(v, w) \in E(G) \Longrightarrow(\phi(v), \phi(w)) \in E(H)$. A graph homomorphism $f$ is called an isomorphism if $f$ is bijective and $f^{-1}$ is also a graph homomorphism. Two graphs are called isomorphic, if there exists an isomorphism between them. If $G$ and $H$ are isomorphic, we write $G \cong H$. The chromatic number $\chi(G)$ of a graph $G$ is defined as $\chi(G):=\min \left\{n \mid \exists\right.$ a graph homomorphism from $G$ to $\left.K_{n}\right\}$. Here, $K_{n}$ denotes a complete graph on $n$ vertices.

Let $G$ be a graph and $v$ be a vertex of $G$. The neighbourhood of $v$ is defined as $N(v)=\{w \in V(G) \mid(v, w) \in E(G)\}$. If $A \subset V(G)$, the set of neighbours of $A$ is defined as $N(A)=\{x \in V(G) \mid(x, a) \in E(G) \forall a \in A\}$. The degree of a vertex $v$ is $|N(v)|$. A graph is said to be $d$-regular, if each vertex has degree $d$. The maximal degree of $G$ is the maximum of the degree of vertices of $G$.

\subsection{Simplicial complex}

A finite abstract simplicial complex $X$ is a collection of finite sets such that if $\tau \in X$ and $\sigma \subset \tau$, then $\sigma \in X$. The elements of $X$ are called simplices of $X$. The dimension of a simplex $\sigma$ is equal to $|\sigma|-1$. The dimension of an abstract simplicial complex is the maximum of the dimensions of its simplices. The 0-dimensional simplices are called vertices of $X$. If $\sigma \subset \tau$, we say that $\sigma$ is a face of $\tau$. If a simplex has dimension $k$, it is said to be $k$-dimensional or $k$-simplex. The boundary of a $k$-simplex $\sigma$ is the simplicial complex, consisting of all faces of $\sigma$ of dimension $\leqslant k-1$ and it is denoted by $B d(\sigma)$. A simplex which is not a face of any other simplex is called a maximal simplex. The set of maximal simplices of $X$ is denoted by $M(X)$. A simplicial complex is called pure $d$-dimensional, if all of its maximal simplices are of dimension $d$.

Let $X$ be a simplicial complex and $\tau, \sigma \in X$ such that $\sigma \subsetneq \tau$ and $\tau$ is the only maximal simplex in $X$ that contains $\sigma$. A simplicial collapse of $X$ is the simplicial complex $Y$ obtained from $X$ by removing all those simplices $\gamma$ of $X$ such that $\sigma \subseteq \gamma \subseteq \tau$. Here, $\sigma$ is called a free face of $\tau$ and $(\sigma, \tau)$ is called a collapsible pair. We denote this collapse by $X \searrow Y$. In particular, if $X \searrow Y$, then $X \simeq Y$.

\subsection{Shellability}

A pure $d$-dimensional simplicial complex $X$ is said to be shellable, if its maximal simplices can be ordered $\Gamma_{1}, \Gamma_{2} \ldots, \Gamma_{t}$ in such a way that the subcomplex $\left(\bigcup_{i=1}^{k-1} \Gamma_{i}\right) \cap \Gamma_{k}$ is 
pure and $(d-1)$-dimensional for all $k=2, \ldots, t$. Here, this ordering $\Gamma_{1}, \ldots, \Gamma_{t}$ of maximal simplices is called a shelling order. A maximal simplex $\Gamma_{k}$ is called spanning with respect to the given shelling order if $B d\left(\Gamma_{k}\right) \subseteq \bigcup_{i=1}^{k-1} \Gamma_{i}$.

From [1, Theorem 12.3] we have the following result which tells us about the homotopy type of shellable complexes.

Proposition 8. Assume that $X$ be a shellable simplicial complex, with $\Gamma_{1}, \Gamma_{2}, \ldots, \Gamma_{t}$ being the corresponding shelling order of the maximal simplices, and $\sum$ being the set of spanning simplices. Then

$$
X \simeq \bigvee_{\sigma \in \sum} S^{\operatorname{dim} \sigma}
$$

Remark 9. Any connected 1-dimensional simplicial complex is always shellable and therefore it is either contractible or homotopy equivalent to wedge sum of circles.

\subsection{Folding}

Let $G$ be a graph and $N(u) \subset N(v)$ for $u, v \in V(G), u \neq v$. In this case, the graph homomorphism $V(G) \rightarrow V(G) \backslash\{u\}$, which sends $u$ to $v$ and fixes all other vertices, called folding and the graph $G \backslash\{u\}$ is called a fold of $G$. Here, $V(G \backslash\{u\})=V(G) \backslash\{u\}$ and the edges in the subgraph $G \backslash\{u\}$ are all those edges of $G$ which do not contain $u$.

Proposition 10. ([1], Proposition 4.2 and Proposition 5.1)

Let $G$ be a graph and $u \in V(G)$. If $G$ is folded on to $G \backslash\{u\}$, then $\mathcal{N}(G)$ is of same homotopy type as $\mathcal{N}(G \backslash\{u\})$.

\section{Proofs}

In this article $[n]$ denotes the set $\{1,2, \ldots, n\}$. Throughout this paper, all the graphs are finite and simple, i.e., not contain loops.

Proof of Theorem 2. Since, by Proposition 10, folding preserve the homotopy type in neighborhood complex, without loss of generality we assume that $G$ cannot be folded onto any of its subgraphs. If the maximal degree of $G$ is 1 , then $G \cong K_{2}$. If the maximal degree of $G$ is 2 , then $\mathcal{N}(G)$ is a 1-dimensional complex and the result follows from Remark 9. So, assume that maximal degree of $G$ is 3 . In this case, $\mathcal{N}(G)$ is 2-dimensional. We show that $\mathcal{N}(G)$ collapses to a 1-dimensional subcomplex. The 2-dimensional simplices of $\mathcal{N}(G)$ are the neighborhoods of vertices of degree 3. Let $\sigma=\{\alpha, \beta, \gamma\}$ be a 2 -simplex of $\mathcal{N}(G)$. Then, there exists $x \in V(G)$ such that $N(x)=\{\alpha, \beta, \gamma\}$. We consider the following two cases.

Case 1. At least one of the $(\alpha, \beta),(\beta, \gamma)$ or $(\alpha, \gamma) \in E(G)$.

Without loss of generality assume that $(\alpha, \beta) \in E(G)$ (see Figure 1c). If $\alpha$ and $\beta$ do not have any common neighbor other than $x$, then $(\{\alpha, \beta\}, \sigma)$ is a collapsible pair and therefore $\mathcal{N}(G) \searrow \mathcal{N}(G) \backslash\{\sigma,\{\alpha, \beta\}\}$. 
Assume $\alpha, \beta$ have a common neighbor $y \neq x$. Since, $G \neq K_{4}, y \neq \gamma$. If $y \sim \gamma$, then $N(y)=\{\alpha, \beta, \gamma\}$ and $(\{\alpha, \beta\}, \sigma)$ is a collapsible pair. If $y \nsim \gamma$, then since $N(\alpha)=$ $\{x, \beta, y\}, N(\beta)=\{x, \alpha, y\}$ and maximal degree of $G$ is 3 , we see that $(\{\beta, \gamma\}, \sigma)$ is a collapsible pair.

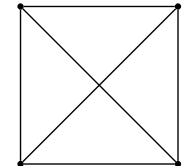

(a) $K_{4}$

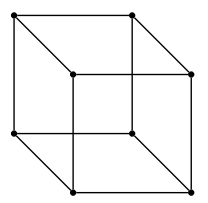

(b) $T$

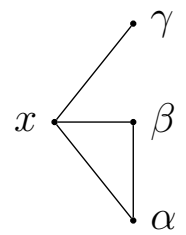

(c)

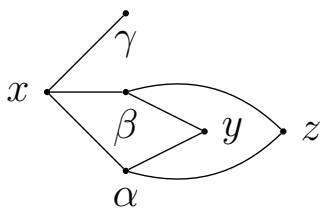

(d)

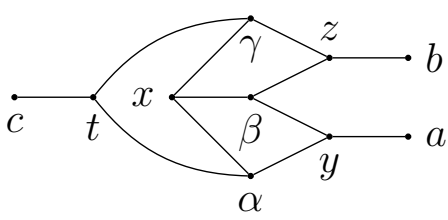

(e)

Figure 1

Case 2. None of the $(\alpha, \beta),(\beta, \gamma)$ or $(\alpha, \gamma) \in E(G)$.

Since maximal degree of $G$ is 3, any 1-dimensional simplex of $\mathcal{N}(G)$ can be a face of at most three 2 -simplices. If there exists a face, say $\{\alpha, \beta\}$ of $\{\alpha, \beta, \gamma\}$ which is a face of three 2-simplices of $\mathcal{N}(G)$ (see Figure 1d), then since $x$ is not contained in any 3-cycle (a graph on 3 vertices, where each vertex has degree 2$), \gamma \neq y, z$. In this case, $(\{\alpha, \gamma\}, \sigma)$ is a collapsible pair.

Assume none of the 1-dimensional face of $\{\alpha, \beta, \gamma\}$ is contained in three maximal simplices, i.e., each face is contained in at most two 2-simplices of $\mathcal{N}(G)$. If some face of $\sigma$ is contained in only one 2 -simplex, then clearly that face will be a free face of $\sigma$. So, assume that each face of $\sigma$ contained in exactly two 2-simplices of $N(G)$ (see Figure 1e). If $y=z=t$, then since maximal degree of $G$ is 3 , at least one of the 1-dimensional face of $\{\alpha, \beta, \gamma\}$ will be a free face.

Suppose exactly two elements of $\{y, z, t\}$ are same, say $y=z$. In this case $\{\beta, \gamma\}$ is a free face of $\{\alpha, \beta, \gamma\}$. Assume $|\{y, z, t\}|=3$. Since, $G$ cannot be folded onto any of its subgraph, degree of $y, z$ and $t$ must be 3 . Let $a, b$ and $c$ are as depicted in Figure 1 e and $\{a, b, c\} \cap\{\alpha, \beta, \gamma\}=\emptyset$.

If $|\{a, b, c\}|=3$, then since the common neighbor of $\gamma$ and $c$ is only $t$, we see that $(\{\gamma, c\}, N(t))$ is a collapsible pair. Hence, $\mathcal{N}(G) \searrow \mathcal{N}(G) \backslash\{N(t),\{\gamma, c\}\}$. Now $(\{\alpha, \gamma\}$, $N(x)$ ) is a collapsible pair in $\mathcal{N}(G) \backslash\{N(t),\{\gamma, c\}\}$ and therefore $\mathcal{N}(G) \searrow \mathcal{N}(G) \backslash\{N(x)$, $N(t),\{\alpha, \gamma\},\{\gamma, c\}\}$.

Now, let $|\{a, b, c\}|=2$. Without loss of generality we can assume that $b=c$. Then $(\{\beta, a\}, N(y))$ is a collapsible pair and therefore $\mathcal{N}(G) \searrow \mathcal{N}(G) \backslash\{N(y),\{\beta, a\}\}$. Now 
$(\{\alpha, \beta\}, N(x))$ is a collapsible pair in $\mathcal{N}(G) \backslash\{N(y),\{\beta, a\}\}$ and therefore $\mathcal{N}(G) \searrow \mathcal{N}(G) \backslash$ $\{N(x), N(y),\{\alpha, \beta\},\{\beta, a\}\}$. If $a=b=c$, then $G \cong T$, which is not possible.

Thus $\mathcal{N}(G)$ collapses to a 1-dimensional subcomplex. From Remark 9, each connected component of $\mathcal{N}(G)$ is either contractible or homotopy equivalent to wedge sum of circles.

Remark 11. It has been shown in [8] that the neighborhood complex of any non-bipartite graph is connected and is never homotopically trivial. Therefore, the nieghborhood complex of any non-bipartite graph of maximal degree at most 3 is homotopy equivalent to a wedge sum of circles.

We now fix some notations. Throughout this article, if we write an integer $r$ as a vertex of $C_{n}(s, t)$, it is understood that we are taking $r$ modulo $n$. Further, for any two vertices $v_{1}$ and $v_{2}$ of $C_{n}(s, t), v_{1}=v_{2}$ means $v_{1} \equiv v_{2}(\bmod n)$. Since $n$ is fixed, for any two integers $x$ and $y$ such that $x \equiv y(\bmod n)$, if no confusion arises, we just write $x \equiv y$.

For any set $X \subset \mathbb{Z}$ and any integer $r$, let $X+r=\{x+r \mid x \in X\}$. Observe that, for any $k \in[n]=V\left(C_{n}(s, t)\right)$, the neighborhood of $k, N(k)=\{s+k, t+k, n-s+k, n-t+k\}$. Since $n \geqslant 5$, if $s, t \neq \frac{n}{2}$, then $C_{n}(s, t)$ is a 4-regular graph, i.e., $|\{s+k, t+k, n-s+k, n-t+k\}|=4$. Since $N(k+r)=N(k)+r$ for any $k, r \in[n]$, observe that any two connected components of $\mathcal{N}\left(C_{n}(s, t)\right)$ are homeomorphic.

Proof of Theorem 3. If $2 s=n$ or $2 t=n$, then $C_{n}(s, t)$ will be a 3-regular graph and the result follows from Theorem 2. Assume $2 s, 2 t \neq n$ and $2(s+t)=n$. We consider the following two cases.

Case 1. $|t-s|=\frac{n}{4}$.

Without loss of generality we can assume that $t>s$ and let $t-s=m$. Then $4 m=n, s+t=2 m, 2 s=m$. Let $k \in[n]$. Now, $N(k+m)=\{s+k+m, t+k+m, n-s+$ $k+m, n-t+k+m\}$ and $N(k)=\{s+k, t+k, n-s+k, n-t+k\}$. Here, $s+k+m=k+t$, $t+k+m=n-t+k, n-s+k+m \equiv m-s+k=s+k$ and $n-t+k+m=n-s+k$. Hence $N(k)=N(k+m)=N(k+2 m)=N(k+3 m)$ for all $k \in[n]$. For any $i, j \in[m], i \neq j$, it can be easily check that $N(i) \cap N(j)=\emptyset$.

Thus $\mathcal{N}\left(C_{n}(s, t)\right)$ is consists of $m$ disjoint simplices of dimension 3 and therefore it is homotopy equivalent to $m$ distinct points.

Case 2. $|t-s| \neq \frac{n}{4}$.

Let $t+s=p$. Then $n=2 p$. Since $n-t+k=n-t-s+s+k=p+s+k, n-s+k=p+t+k$, we see that $N(k)=N(k+p)$ for all $k \in[n]$. Since $N(s+k)=\{2 s+k, s+t+k, k, 2 s+p+k\}$ and $N(t+k)=\{s+t+k, 2 t+k, 2 t+p+k, k\}, N(s+k) \cap[p]=N(t+k) \cap[p]$ implies that $\{2 s+k, 2 s+p+k\} \cap[p]=\{2 t+k, 2 t+p+k\} \cap[p]$. Since $s \neq t, 2 s+k \not \equiv 2 t+k$. However, $2 s+k \equiv 2 t+p+k$ implies that $3 t \equiv s$. Since $t, s<\frac{n}{2}, 3 t=s$ or $3 t=s+n$. If $3 t=s$, then $p=4 t$. But, then $s-t=2 t=\frac{p}{2}=\frac{n}{4}$, which is a contradiction. If $3 t=s+n=s+2 t+2 s$, then $3 s=t$ and $p=4 s$. Here, $t-s=2 s=\frac{p}{2}=\frac{n}{4}$, which is not possible. By an argument similar as above, $2 s+p+k \notin\{2 t+k, 2 t+p+k\}$. Hence $N(s+k) \cap[p] \neq N(t+k) \cap[p] \forall k \in[n]$. 
Since $N(s+k)=N(p+s+k)=N(n-t+k), N(t+k)=N(p+t+k)=N(n-s+k)$, we conclude that each vertex $k$ belongs to exactly two maximal simplices, namely $N(s+k)$ and $N(t+k)$. Further, $N(i) \cap[p] \neq N(j) \cap[p] \forall 1 \leqslant i \neq j \leqslant p$.

Observe that, $|N(k) \cap\{1, \ldots, p\}|=2$ for all $k \in[n]$. Since $N(k)=N(k+p)$ for all $k \in$ $[n], C_{n}(s, t)$ folded onto the induced subgraph $C_{n}(s, t)[\{1, \ldots, p\}]$. Hence $\mathcal{N}\left(C_{n}(s, t)\right) \simeq$ $\mathcal{N}\left(C_{n}(s, t)[\{1, \ldots, p\}]\right)$, by Proposition 10. Since each vertex $k$ of $C_{n}(s, t)$ belongs to exactly two maximal simplices $N(s+k)=N(p+s+k)$ and $N(t+k)=N(p+t+k)$ of $\mathcal{N}\left(C_{n}(s, t)\right)$, and $N(i) \cap[p] \neq N(j) \cap[p] 1 \leqslant i \neq j \leqslant p$, we see that each vertex $x \in[p]$ also belongs to exactly two maximal simplices of $\mathcal{N}\left(C_{n}(s, t)[\{1, \ldots, p\}]\right)$. Further, since $\mathcal{N}\left(C_{n}(s, t)[\{1, \ldots, p\}]\right)$ is a 1-dimensional complex, the connected components of $\mathcal{N}\left(C_{n}(s, t)[\{1, \ldots, p\}]\right)$ cannot be contractible. The result follows from Remark 9.

Proof of Theorem $4(A)$. Let $3 s=t$ and $n=10 s$. For each $1 \leqslant i \leqslant s$, let $G_{i}$ be the subgraph of $C_{n}(s, t)$ induced by the vertex set $\{i, i+s, i+2 s, i+3 s, i+4 s, i+5 s, i+6 s, i+$ $7 s, i+8 s, i+9 s\}$. Observe that each $G_{i}$ is isomorphic to $C_{10}(1,3)$ and $C_{n}(s, t) \cong \bigsqcup_{i=1}^{s} G_{i}$. Therefore $\mathcal{N}\left(C_{n}(s, t)\right) \cong \bigsqcup_{i=1}^{s} \mathcal{N}\left(C_{10}(1,3)\right)$. It can be easily verified that $\mathcal{N}\left(C_{10}(1,3)\right)$ is homeomorphic to disjoint union of two copies of simplicial boundary of a 4-simplex, namely the subcomplex $N(1) \cup N(3) \cup N(5) \cup N(7) \cup N(9)=B d(\{2,4,6,8,10\})$ and the subcomplex $N(2) \cup N(4) \cup N(6) \cup N(8) \cup N(10)=B d(\{1,3,5,7,9\})$.

We conclude that $\mathcal{N}\left(C_{n}(s, t)\right) \cong \bigsqcup_{2 s \text {-copies }} S^{3}$. The case $3 t=s$ and $n=10 t$, follows by symmetry.

Proof of Theorem $4(B)$. Let $3 s=t$ and $n=12 s$. For each $1 \leqslant i \leqslant s$, let $G_{i}$ be the subgraph of $C_{n}(s, t)$ induced by the vertex set $\{i, i+s, i+2 s, i+3 s, i+4 s, i+5 s, i+$ $6 s, i+7 s, i+8 s, i+9 s, i+10 s, i+11 s\}$. Observe that each $G_{i}$ is isomorphic to $C_{12}(1,3)$ and $C_{n}(s, t) \cong \bigsqcup_{i=1}^{s} G_{i}$. We now compute $\mathcal{N}\left(C_{12}(1,3)\right)$.

For each $i \in\{1,2\}$, let $A_{i}$ be the subcomplex of $\mathcal{N}\left(C_{12}(1,3)\right)$, where the set of maximal simplices $M\left(A_{i}\right)=\{N(i), N(i+2), N(i+4), N(i+6), N(i+8), N(i+10)\}$. Then $\mathcal{N}\left(C_{12}(1,3)\right)=A_{1} \sqcup A_{2}$. It can be easily checked that for each $k,(\{k+1, k+3, k+9\}, N(k))$ is a collapsible pair in $\mathcal{N}\left(C_{12}(1,3)\right)$ and therefore $N(k) \searrow \tau_{k}^{1}=\{k+1, k+3, k+11\}, \tau_{k}^{2}=$ $\{k+3, k+9, k+11\}$ and $\tau_{k}^{3}=\{k+1, k+9, k+11\}$. Since, $\tau_{k+10}^{1}=\{k+11, k+1, k+9\}=\tau_{k}^{3}$ for all $k \in[12]$, we see that $\mathcal{N}\left(C_{12}(1,3)\right)$ collapses to a subcomplex $\Delta$, where the set of maximal simplices $M(\Delta)=\left\{\tau_{k}^{i} \mid i \in\{1,2\}, k \in[12]\right\}$. For, $i \in\{1,2\}$, let $\Delta_{i}=\Delta \cap A_{i}$. Then, $\Delta=\Delta_{1} \sqcup \Delta_{2}$. It can be easily verified that each $\Delta_{i}$ is a shellable complex and the shelling order is given by

$$
\tau_{i}^{1}, \tau_{i}^{2}, \tau_{i+2}^{1}, \tau_{i+2}^{2}, \tau_{i+4}^{1}, \tau_{i+4}^{2}, \tau_{i+6}^{2}, \tau_{i+6}^{1}, \tau_{i+8}^{1}, \tau_{i+8}^{2}, \tau_{i+10}^{1}, \tau_{i+10}^{2}
$$

Here, $\tau_{i+8}^{2}$ and $\tau_{i+10}^{2}$ are spanning simplices with respect to the shelling order 1 . Hence, $\Delta_{i} \simeq S^{2} \vee S^{2}$ and by Proposition 8. We conclude that $\mathcal{N}\left(C_{n}(s, t)\right) \cong \bigsqcup_{2 s-\text { copies }} S^{2} \vee S^{2}$.

If $3 t=s$ and $n=12 t$, the result follows by symmetry. 
Proof of Theorem $4(C)$. Let $3 s=t$ and $8 s, 10 s, 12 s \neq n$. Observe that, $2 s, 4 s, 6 s, 8 s$, $12 s \not \equiv 0$. For each $k \in[n], N(k)=\{s+k, 3 s+k, n-s+k, n-3 s+k\}$. Since $4 s, 6 s, 8 s, 10 s$, $12 s \not \equiv 0$, we see that $N(3 s+k) \cap N(n-3 s+k)=\{k\}$ and therefore $(\{3 s+k, n-$ $3 s+k\}, N(k))$ is a collapsible pair. Hence, $N(k) \searrow \tau_{k}^{1}=\{3 s+k, s+k, n-s+k\}$ and $\tau_{k}^{2}=\{n-3 s+k, s+k, n-s+k\} \forall k \in[n]$. Since $\tau_{n-2 s+k}^{1}=\{s+k, n-s+k, n-3 s+k\}=\tau_{k}^{2}$, $\mathcal{N}\left(C_{n}(s, t)\right)$ collapses to a subcomplex $\Delta$, where $M(\Delta)=\left\{\tau_{k}^{1} \mid k \in[n]\right\}$.

Using the fact that $2 s, 4 s, 6 s, 8 s, 12 s \not \equiv 0$, it can be easily check that $N(3 s+k) \cap$ $N(n-s+k)=\{2 s+k, k\}$. Since $\{3 s+k, n-s+k\} \nsubseteq \tau_{2 s+k}^{1}=\{5 s+k, 3 s+k, s+k\}$, we conclude that $\left(\{3 s+k, n-s+k\}, \tau_{k}^{1}\right)$ is a collapsible pair. Hence, for each $k \in[n]$, $\tau_{k}^{1} \searrow \delta_{k}^{1}=\{3 s+k, s+k\}$ and $\delta_{k}^{2}=\{n-s+k, s+k\}$. Since $\delta_{n-2 s+k}^{1}=\{s+k, n-s+k\}=\delta_{k}^{2}$. Applying the collapsible pairs of the type $\left(\{3 s+k, n-s+k\}, \tau_{k}^{1}\right)$ for each $k \in[n], \Delta$ collapses to a 1-dimensional subcomplex $\Delta^{\prime}$, where $M\left(\Delta^{\prime}\right)=\left\{\delta_{k}^{1} \mid k \in[n]\right\}$.

Since each vertex $k \in[n]$ belongs to $\delta_{n-s+k}^{1}$ and $\delta_{n-3 s+k}^{1}$, and $\delta_{n-s+k}^{1} \neq \delta_{n-3 s+k}^{1}$, connected components of $\Delta^{\prime}$ cannot be contractible. Therefore, by Proposition 8 , each connected component of $\Delta^{\prime}$ is homotopy equivalent to wedge sum of circles.

Proof of Theorem 5(A). Let $5 s=3 t$ and $n=4 t$. Since $s \neq t, n \neq 4 s$. Further, since $3 s+t=n$ implies that $s=t$ and $3 s-t=n$ implies that $3 s=5 t=\frac{25}{3} s$, we see that $3 s+t, 3 s-t \neq n$. The result follows from Theorem 13 . The case $5 t=3 s$ and $n=4 s$, follows by symmetry.

Proof of Theorem 5(B). Let $5 s=3 t$ and $n=4 s$. Since 5 and 3 are relatively prime, there exists $s^{\prime}$ such that $s=3 s^{\prime}$ and $t=5 s^{\prime}$. Here, $n=12 s^{\prime}$ and $N(k)=\left\{3 s^{\prime}+k, 5 s^{\prime}+\right.$ $\left.k, 9 s^{\prime}+k, 7 s^{\prime}+k\right\} \forall k \in[n]$. For $i \in\left\{1, \ldots, 2 s^{\prime}\right\}$, let $B_{i}$ be the subcomplex of $\mathcal{N}\left(C_{n}(s, t)\right)$, where $M\left(B_{i}\right)=\left\{N\left(i+2 s^{\prime} l\right) \mid l \in\{0,1,2,3,4,5\}\right\}$. It can be easily checked that the set of vertices of $B_{i}$ is given by $V\left(B_{i}\right)=\left\{i+s^{\prime}, i+3 s^{\prime}, i+5 s^{\prime}, i+7 s^{\prime}, i+9 s^{\prime}, i+11 s^{\prime}\right\}$ and $V\left(B_{l}\right) \cap V\left(B_{m}\right)=\emptyset \forall 1 \leqslant l \neq m \leqslant 2 s^{\prime}$. Hence $B_{l} \cap B_{m}=\emptyset \forall 1 \leqslant l \neq m \leqslant 2 s^{\prime}$.

Let $k \in[n]$. Since $N\left(3 s^{\prime}+k\right) \cap N\left(5 s^{\prime}+k\right) \cap N\left(9 s^{\prime}+k\right)=\{k\},\left(\left\{3 s^{\prime}+k, 5 s^{\prime}+k, 9 s^{\prime}+\right.\right.$ $k\}, N(k))$ is a collapsible pair. Therefore $N(k) \searrow \tau_{k}^{1}=\left\{3 s^{\prime}+k, 9 s^{\prime}+k, 7 s^{\prime}+k\right\}, \tau_{k}^{2}=$ $\left\{5 s^{\prime}+k, 9 s^{\prime}+k, 7 s^{\prime}+k\right\}$ and $\tau_{k}^{3}=\left\{3 s^{\prime}+k, 5 s^{\prime}+k, 7 s^{\prime}+k\right\}$. Since, $\tau_{n-2 s^{\prime}+k}^{2}=\left\{3 s^{\prime}+\right.$ $\left.k, 7 s^{\prime}+k, 5 s^{\prime}+k\right\}=\tau_{k}^{3}$, we conclude that $\mathcal{N}\left(C_{n}(s, t)\right)$ collapses to a subcomplex $\Delta$, where $M(\Delta)=\left\{\tau_{k}^{i} \mid i \in\{1,2\}, k \in[n]\right\}$.

For $i \in\left\{1, \ldots, 2 s^{\prime}\right\}$, let $\Delta_{i}=\Delta \cap B_{i}$. Since $B_{i} \cap B_{j}=\emptyset, \Delta_{i} \cap \Delta_{j}=\emptyset \forall 1 \leqslant i \neq j \leqslant 2 s^{\prime}$. Further, $M\left(\Delta_{i}\right)=\left\{\tau_{i+2 s^{\prime} l}^{j} \mid j \in\{1,2\}, l \in\{0,1,2,3,4,5\}\right\} \forall 1 \leqslant i \leqslant 2 s^{\prime}$.

We now show that each $\Delta_{i}$ is a shellable complex and the shelling order is given by

$$
\tau_{i}^{1}, \tau_{i}^{2}, \tau_{i+2 s^{\prime}}^{1}, \tau_{i+2 s^{\prime}}^{2}, \tau_{i+4 s^{\prime}}^{1}, \tau_{i+4 s^{\prime}}^{2}, \tau_{i+6 s^{\prime}}^{1}, \tau_{i+6 s^{\prime}}^{2}, \tau_{i+8 s^{\prime}}^{1}, \tau_{i+8 s^{\prime}}^{2}, \tau_{i+10 s^{\prime}}^{1}, \tau_{i+10 s^{\prime}}^{2} .
$$

For each $i \in[n]$ and $0 \leqslant l \leqslant 5$, let $\tau_{i+2 l s^{\prime}}=\tau_{i}^{1} \cup \tau_{i}^{2} \cup \ldots \cup \tau_{i+2 l s^{\prime}}^{1} \cup \tau_{i+2 l s^{\prime}}^{2}$. The following are easy to verify.

$$
\tau_{i}^{1}=\left\{3 s^{\prime}+i, 9 s^{\prime}+i, 7 s^{\prime}+i\right\}, \tau_{i}^{2}=\left\{5 s^{\prime}+i, 9 s^{\prime}+i, 7 s^{\prime}+i\right\} \text { and } M\left(\tau_{i}^{1} \cap \tau_{i}^{2}\right)=\left\{\left\{9 s^{\prime}+\right.\right.
$$
$\left.\left.i, 7 s^{\prime}+i\right\}\right\}$.

$\tau_{i+2 s^{\prime}}^{1}=\left\{5 s^{\prime}+i, 11 s^{\prime}+i, 9 s^{\prime}+i\right\}$ and $M\left(\tau_{i} \cap \tau_{i+2 s^{\prime}}^{1}\right)=\left\{\left\{5 s^{\prime}+i, 9 s^{\prime}+i\right\}\right\}$.

$\tau_{i+2 s^{\prime}}^{2}=\left\{7 s^{\prime}+i, 11 s^{\prime}+i, 9 s^{\prime}+i\right\}$ and $M\left(\left(\tau_{i} \cup \tau_{i+2 s^{\prime}}^{1}\right) \cap \tau_{i+2 s^{\prime}}^{2}\right)=\left\{\left\{11 s^{\prime}+i, 9 s^{\prime}+i\right\},\left\{9 s^{\prime}+\right.\right.$ $\left.\left.i, 7 s^{\prime}+i\right\}\right\}$. 
$\tau_{i+4 s^{\prime}}^{1}=\left\{7 s^{\prime}+i, s^{\prime}+i, 11 s^{\prime}+i\right\}$ and $M\left(\tau_{i+2 s^{\prime}} \cap \tau_{i+4 s^{\prime}}^{1}\right)=\left\{\left\{7 s^{\prime}+i, 11 s^{\prime}+i\right\}\right\}$.

$\tau_{i+4 s^{\prime}}^{2}=\left\{9 s^{\prime}+i, s^{\prime}+i, 11 s^{\prime}+i\right\}$ and $M\left(\left(\tau_{i+2 s^{\prime}} \cup \tau_{i+4 s^{\prime}}^{1}\right) \cap \tau_{i+4 s^{\prime}}^{2}\right)=\left\{\left\{s^{\prime}+i, 11 s^{\prime}+\right.\right.$ $\left.i\},\left\{9 s^{\prime}+i, 11 s^{\prime}+i\right\}\right\}$.

$\tau_{i+6 s^{\prime}}^{1}=\left\{9 s^{\prime}+i, 3 s^{\prime}+i, s^{\prime}+i\right\}$ and $M\left(\tau_{i+4 s^{\prime}} \cap \tau_{i+6 s^{\prime}}^{1}\right)=\left\{\left\{3 s^{\prime}+i, 9 s^{\prime}+i\right\},\left\{s^{\prime}+i, 9 s^{\prime}+i\right\}\right\}$.

$\tau_{i+6 s^{\prime}}^{2}=\left\{11 s^{\prime}+i, 3 s^{\prime}+i, s^{\prime}+i\right\}$ and $M\left(\left(\tau_{i+4 s^{\prime}} \cup \tau_{i+6 s^{\prime}}^{1}\right) \cap \tau_{i+6 s^{\prime}}^{2}\right)=\left\{\left\{11 s^{\prime}+i, s^{\prime}+\right.\right.$ $\left.i\},\left\{3 s^{\prime}+i, s^{\prime}+i\right\}\right\}$.

$\tau_{i+8 s^{\prime}}^{1}=\left\{11 s^{\prime}+i, 5 s^{\prime}+i, 3 s^{\prime}+i\right\}$ and $M\left(\tau_{i+6 s^{\prime}} \cap \tau_{i+8 s^{\prime}}^{1}\right)=\left\{\left\{11 s^{\prime}+i, 3 s^{\prime}+i\right\},\left\{11 s^{\prime}+\right.\right.$ $\left.\left.i, 5 s^{\prime}+i\right\}\right\}$.

$\tau_{i+8 s^{\prime}}^{2}=\left\{s^{\prime}+i, 5 s^{\prime}+i, 3 s^{\prime}+i\right\}$ and $M\left(\left(\tau_{i+6 s^{\prime}} \cup \tau_{i+8 s^{\prime}}^{1}\right) \cap \tau_{i+8 s^{\prime}}^{2}\right)=\left\{\left\{s^{\prime}+i, 3 s^{\prime}+i\right\},\left\{5 s^{\prime}+\right.\right.$ $\left.\left.i, 3 s^{\prime}+i\right\}\right\}$.

$\tau_{i+10 s^{\prime}}^{1}=\left\{s^{\prime}+i, 7 s^{\prime}+i, 5 s^{\prime}+i\right\}$ and $M\left(\tau_{i+8 s^{\prime}} \cap \tau_{i+10 s^{\prime}}^{1}\right)=B d\left(\tau_{i+10 s^{\prime}}^{1}\right)$.

$\tau_{i+10 s^{\prime}}^{2}=\left\{3 s^{\prime}+i, 7 s^{\prime}+i, 5 s^{\prime}+i\right\}$ and $M\left(\left(\tau_{i+8 s^{\prime}} \cup \tau_{i+10 s^{\prime}}^{1}\right) \cap \tau_{i+10 s^{\prime}}^{2}\right)=B d\left(\tau_{i+10 s^{\prime}}^{2}\right)$.

Thus the order given in (2) is a shelling order and the spanning simplices are $\tau_{i+10 s^{\prime}}^{1}$ and $\tau_{i+10 s^{\prime}}^{2}$. The result follows by Proposition 8 . The case $5 t=3 s$ and $n=4 t$, follows by symmetry.

We need the following lemma to prove Theorem 5 (C).

Lemma 12. Let $s, t \in\{1, \ldots, n-1\}$ such that $2 s, 2(s+t), 3 s+t, 3 s-t, 4 s \not \equiv 0(\bmod n)$. Then for each $k \in[n],(\{s+k, n-s+k\}, N(k))$ is a collapsible pair in $\mathcal{N}\left(C_{n}(s, t)\right)$.

Proof. If there exists $x \in[n], x \neq k$ such that $\{s+k, n-s+k\} \subset N(x)$, then $x \in$ $\{2 s+k, s+t+k, n-t+s+k\} \cap\{n-s+t+k, n-2 s+k, n-t-s+k\}$.

Since, $0 \not \equiv 3 s-t, 2 s+k \neq n-s+t+k$. Further, since $2 s+k=n-2 s+k$ implies that $4 s \equiv 0$ and $2 s+k=n-t-s+k$ implies that $3 s+t \equiv 0$, we conclude that $x \neq 2 s+k$.

$s+t+k=n-s+t+k \Longrightarrow 2 s \equiv 0$ and $s+t+k=n-2 s+k \Longrightarrow 3 s+t \equiv 0$. Further, since $s+t+k=n-t-s+k \Longrightarrow 0 \equiv 2(s+t), x \neq s+t+k$.

Since, $s \neq t$ and $3 s-t \not \equiv 0$, we conclude that $n-t+s+k \neq n-s+t+k, n-2 s+$ $k, n-t-s+k$.

Thus there exists no $x \in[n]$ different from $k$ such that $\{s+k, n-s+k\} \subset N(x)$ and therefore $(\{s+k, n-s+k\}, N(k))$ is a collapsible pair.

Proof of Theorem $5(C)$. Let $5 s=3 t$ and one of $6 s$ or $\frac{14 s}{3}$ is equal to $n$. There exists an integer $s^{\prime}$ such that $s=3 s^{\prime}$ and $t=5 s^{\prime}$.

Case 1. $n=6 s$.

In this case $n=18 s^{\prime}$ and $N(k)=\left\{3 s^{\prime}+k, 5 s^{\prime}+k, 13 s^{\prime}+k, 15 s^{\prime}+k\right\}$ for all $k \in[n]$. Since $3 s-t=n \Longrightarrow \frac{4 s}{3}=n$ and $3 s+t=n \Longrightarrow \frac{14 s}{3}=n$, we see that $3 s-t, 3 s+t \neq n$. Further, $s, t<\frac{n}{2}$ implies that $3 s-t, 3 s+t \not \equiv n$. Using Lemma 12, $N(k) \searrow \tau_{k}^{1}=$ $\left\{3 s^{\prime}+k, 5 s^{\prime}+k, 13 s^{\prime}+k\right\}$ and $\tau_{k}^{2}=\left\{15 s^{\prime}+k, 5 s^{\prime}+k, 13 s^{\prime}+k\right\}$ for all $k \in[n]$. Since, $\tau_{k+10 s^{\prime}}^{1}=\left\{13 s^{\prime}+k, 15 s^{\prime}+k, 5 s^{\prime}+k\right\}=\tau_{k}^{2}, \mathcal{N}\left(C_{n}(s, t)\right)$ collapses to a subcomplex $\Delta$, where $M(\Delta)=\left\{\tau_{k}^{1} \mid k \in[n]\right\}$.

Let $k \in[n]$. Observe that $N\left(3 s^{\prime}+k\right) \cap N\left(5 s^{\prime}+k\right)=\left\{k, 8 s^{\prime}+k\right\}$ and $\left\{3 s^{\prime}+k, 5 s^{\prime}+k\right\} \nsubseteq$ $\tau_{8 s^{\prime}+k}^{1}$. Hence $\left(\left\{3 s^{\prime}+k, 5 s^{\prime}+k\right\}, \tau_{k}^{1}\right)$ is a collapsible pair in $\Delta$ and therefore $\tau_{k}^{1} \searrow \delta_{k}^{1}=$ $\left\{3 s^{\prime}+k, 13 s^{\prime}+k\right\}$ and $\delta_{k}^{2}=\left\{5 s^{\prime}+k, 13 s^{\prime}+k\right\}$. Since $\delta_{k+10 s^{\prime}}^{1}=\left\{13 s^{\prime}+k, 5 s^{\prime}+k\right\}=\delta_{k}^{2}$, 
we see that $\Delta$ collapses to a 1-dimensional subcomplex $\Delta^{\prime}$, with $M\left(\Delta^{\prime}\right)=\left\{\delta_{k}^{1} \mid k \in[n]\right\}$. Each vertex $x \in[n]$, belongs to $\delta_{5 s^{\prime}+x}^{1}=\left\{8 s^{\prime}+x, x\right\}$ and $\delta_{15 s^{\prime}+x}^{1}=\left\{x, 10 s^{\prime}+x\right\}$. Since $\delta_{5 s^{\prime}+x}^{1} \neq \delta_{15 s^{\prime}+x}^{1}$, connected components of $\Delta^{\prime}$ cannot be contractible. Result follows from Remark 9.

Case 2. $n=\frac{14 s}{3}$.

In this case $n=14 s^{\prime}$ and $N(k)=\left\{3 s^{\prime}+k, 5 s^{\prime}+k, 11 s^{\prime}+k, 9 s^{\prime}+k\right\} \forall k \in[n]$. Since $3 t-s=n \Longrightarrow 4 s=n$ and $3 t+s=n \Longrightarrow 6 s=n$, we see that $3 t-s, 3 t+s \neq n$. Further, since $4 t \neq n$, by Lemma $12,\left(\left\{5 s^{\prime}+k, 9 s^{\prime}+k\right\}, N(k)\right)$ is a collapsible pair and therefore $N(k) \searrow \tau_{k}^{1}=\left\{5 s^{\prime}+k, 3 s^{\prime}+k, 11 s^{\prime}+k\right\}$ and $\tau_{k}^{2}=\left\{9 s^{\prime}+k, 3 s^{\prime}+k, 11 s^{\prime}+k\right\}$. Since, $\tau_{k+6 s^{\prime}}^{1}=\left\{11 s^{\prime}+k, 9 s^{\prime}+k, 3 s^{\prime}+k\right\}=\tau_{k}^{2}$, we conclude that $\mathcal{N}\left(C_{n}(s, t)\right)$ collapses to a subcomplex $\Delta$, where $M(\Delta)=\left\{\tau_{k}^{1} \mid k \in[n]\right\}$.

$N\left(3 s^{\prime}+k\right) \cap N\left(5 s^{\prime}+k\right)=\left\{k, 8 s^{\prime}+k\right\}$ and $\left\{3 s^{\prime}+k, 5 s^{\prime}+k\right\} \nsubseteq \tau_{8 s^{\prime}+k}^{1}$ implies that $\left(\left\{3 s^{\prime}+k, 5 s^{\prime}+k\right\}, \tau_{k}^{1}\right)$ is a collapsible pair in $\Delta$ and therefore $\tau_{k}^{1} \searrow \delta_{k}^{1}=\left\{3 s^{\prime}+k, 11 s^{\prime}+k\right\}$ and $\delta_{k}^{2}=\left\{5 s^{\prime}+k, 11 s^{\prime}+k\right\}$. Further, since $\delta_{8 s^{\prime}+k}^{1}=\left\{11 s^{\prime}+k, 5 s^{\prime}+k\right\}=\delta_{k}^{2}$, we conclude that $\Delta$ collapses to a 1-dimensional subcomplex $\Delta^{\prime}$, where $M\left(\Delta^{\prime}\right)=\left\{\delta_{k}^{1} \mid k \in[n]\right\}$.

Each vertex $x \in[n]$, belongs to $\delta_{3 s^{\prime}+x}^{1}=\left\{6 s^{\prime}+x, x\right\}$ and $\delta_{11 s^{\prime}+x}^{1}=\left\{x, 8 s^{\prime}+x\right\}$. Since $\delta_{3 s^{\prime}+x}^{1} \neq \delta_{11 s^{\prime}+x}^{1}$, the result follows from Remark 9 .

If $5 t=3 s$ and one of $6 t$ or $\frac{14 t}{3}$ is equal to $n$, the result follows by symmetry.

Proof of Theorem 5(D). Let $5 s=3 t$ and $n \neq 4 s, 4 t, 6 s, \frac{14 s}{3}$. Now, $3 s-t=n \Longrightarrow \frac{4 s}{3}=n$, which is not possible as $s<\frac{n}{2}$. Further, since $3 s+t=n \Longrightarrow \frac{14 s}{3}=n, 3 t+s=n \Longrightarrow$ $6 s=n$ and $3 t-s=n \Longrightarrow 4 s=n$, we see that $3 s-t, 3 s+t, 3 t+s, 3 t-s \neq n$. The result follows from Theorem 14 . The case $5 t=3 s$ and $n \neq 4 s, 4 t, 6 t, \frac{14 t}{3}$, follows by symmetry.

Proof of Theorem 6(A). Let us first assume that one of the $3 s-t, 3 t-s, 3 s+t$ or $3 t+s$ is equal to $n$.

Case 1. $3 t-s=n$ or $3 s-t=n$.

Assume that $3 t-s=n$. We consider the following two cases.

(i) $2 s \neq t$.

In this case, $3 s+t, 3 s-t, 4 s \not \equiv n$. By Lemma 12, $N(k) \searrow \tau_{k}^{1}=\{s+k, t+k, n-t+k\}$ and $\tau_{k}^{2}=\{n-s+k, t+k, n-t+k\}$ for all $k \in[n]$. Further, since $\tau_{k}^{2}=\tau_{t-s+k}^{1}$, $\mathcal{N}\left(C_{n}(s, t)\right)$ collapses to a subcomplex $\Delta$, with $M(\Delta)=\left\{\tau_{i}^{1} \mid i \in[n]\right\}$. It can be easily checked that $N(s+k) \cap N(n-t+k)=\{k, 2 t+k\}$. Since, $\{s+k, n-t+k\} \nsubseteq$ $\tau_{2 t+k}^{1}, \tau_{k}^{1} \searrow \delta_{k}^{1}=\{s+k, t+k\}$ and $\delta_{k}^{2}=\{n-t+k, t+k\}$ for all $k \in[n]$. Now, $\delta_{t-s+k}^{1}=\{t+k, n-t+k\}=\delta_{k}^{2}$ implies that $\Delta$ collapses to a 1-dimensional subcomplex $\Delta^{\prime}$, where $M\left(\Delta^{\prime}\right)=\left\{\delta_{i}^{1} \mid i \in[n]\right\}$.

Each vertex $k \in[n]$ can belongs to only $\delta_{j}^{1}$ for $j \in\{s+k, t+k, n-s+k, n-t+k\}$. Since, $\delta_{s+k}^{1}=\{2 s+k, s+t+k\}, \delta_{t+k}^{1}=\{s+t+k, 2 t+k\}, \delta_{n-s+k}^{1}=\{k, n-s+t+$ $k\}, \delta_{n-t+k}^{1}=\{n+s-t+k, k\}$, we observe that $k$ belongs to only $\delta_{n-s+k}^{1}$ and $\delta_{n-t+k}^{1}$. Further, since $\delta_{n-s+k}^{1} \neq \delta_{n-t+k}^{1}$ and $\Delta^{\prime}$ is a 1-dimensional complex, each connected component of $\Delta^{\prime}$ is homotopy equivalent to $S^{1}$. 
(ii) $2 s=t$.

In this case, $n=5 s$ and $N(k)=\{s+k, 2 s+k, 3 s+k, 4 s+k\} \forall k \in[n]$. For $i \in[n]$, let $\Gamma_{i}:=\{i, s+i(\bmod n), 2 s+i(\bmod n), 3 s+i(\bmod n), 4 s+i(\bmod n)\}$ be a 4 -simplex. It can be easily checked that $\Gamma_{i} \cap \Gamma_{j}=\emptyset \forall 1 \leqslant i \neq j \leqslant s$. The subcomplex of $\mathcal{N}\left(C_{n}(s, t)\right)$ induced by the vertices $k, k+s, k+2 s, k+3 s$ and $k+4 s$, which is equal to $N(k) \cup N(k+s) \cup N(k+2 s) \cup N(k+3 s) \cup N(k+4 s)$ is $\operatorname{Bd}\left(\Gamma_{k}\right)$. We conclude that $\mathcal{N}\left(C_{n}(s, t)\right) \cong \bigsqcup_{k \in\{1,2, \ldots, s\}} B d\left(\Gamma_{k}\right)$. Since $B d\left(\Gamma_{k}\right)$ is homeomorphic to $S^{3}$, the result follows.

The case $3 s-t=n$ follows from symmetry.

Case 2. $3 s-t, 3 t-s \neq n$, i.e., $3 t+s=n$ or $3 s+t=n$.

Assume that $3 t+s=n$.

(i) $2 t \neq s$.

In this case, $3 s+t, 3 s-t, 4 s \not \equiv n$. From Lemma 12, $N(k) \searrow \tau_{k}^{1}=\{s+k, t+k, n-t+k\}$ and $\tau_{k}^{2}=\{n-s+k, t+k, n-t+k\}$ for all $k \in[n]$. Further, since $\tau_{k}^{2}=\tau_{2 t+k}^{1}$, $\mathcal{N}\left(C_{n}(s, t)\right)$ collapses to a subcomplex $\Delta$, where $M(\Delta)=\left\{\tau_{k}^{1} \mid k \in[n]\right\}$.

It can be easily verified that $N(s+k) \cap N(t+k)=\{k, s+t+k\}$. Since, $\{s+k, t+k\} \nsubseteq$ $\tau_{s+t+k}^{1}=\{2 s+t+k, s+2 t+k, s+k\}, \tau_{k}^{1} \searrow \delta_{k}^{1}=\{s+k, n-t+k\}$ and $\delta_{k}^{2}=\{t+k, n-$ $t+k\}$ for all $k \in[n]$. Further, since $\delta_{2 t+k}^{1}=\{2 t+s+k, t+k\}=\{n-t+k, t+k\}=\delta_{k}^{2}$, $\Delta$ collapses to a 1-dimensional subcomplex $\Delta^{\prime}$, where $M\left(\Delta^{\prime}\right)=\left\{\delta_{i}^{1} \mid i \in[n]\right\}$.

Each vertex $k \in[n]$ can belong to only $\delta_{j}^{1}$ for $j \in\{s+k, t+k, n-s+k, n-t+k\}$. Since, $\delta_{s+k}^{1}=\{2 s+k, n-t+s+k\}, \delta_{t+k}^{1}=\{s+t+k, k\}, \delta_{n-s+k}^{1}=\{k, 2 t+k\}$ and $\delta_{n-t+k}^{1}=\{n-t+s+k, t+s+k\}$, we conclude that $k$ belongs to only $\delta_{t+k}^{1}$ and $\delta_{n-s+k}^{1}$. Since $\Delta^{\prime}$ is a 1 -dimensional complex, each connected component of $\Delta$ is homotopy equivalent to $S^{1}$.

(ii) $2 t=s$.

Since $n=3 t+s$ and $s=2 t, n=5 t$. For each $k \in[n]$, let $\Gamma_{k}:=\{k, t+k(\bmod n), 2 t+$ $k(\bmod n), 3 t+k(\bmod n), 4 t+k(\bmod n)\}$ be a $4-$ simplex. It can be easily verified that $\Gamma_{i} \cap \Gamma_{j}=\emptyset \forall 1 \leqslant i \neq j \leqslant t$ and the subcomplex of $\mathcal{N}\left(C_{n}(s, t)\right)$ induced by the vertices $k, k+t, k+2 t, k+3 t$ and $k+4 t$, is equal to $N(k) \cup N(k+t) \cup N(k+3 t) \cup$ $N(k+3 t) \cup N(k+4 t)=B d\left(\Gamma_{k}\right)$. We conclude that $\mathcal{N}\left(C_{n}(s, t)\right) \cong \bigsqcup_{k \in\{1,2, \ldots, t\}} B d\left(\Gamma_{k}\right)$.

The case $3 s+t=n$ follows by symmetry.

Proof of Theorem 6 (B) follows from Theorem 13 and Proof of Theorem 6 (C) follows from Theorem 14.

Theorem 13. Let $s, t \in\left\{1,2, \ldots,\left\lfloor\frac{n}{2}\right\rfloor\right\}$ such that $2 s, 2 t, 2(s+t), 3 s+t, 3 s-t, 4 s \neq n$, $3 s \neq t, 3 t \neq s$. If $4 t=n$, then each connected component of $\mathcal{N}\left(C_{n}(s, t)\right)$ is homotopy equivalent to a garland of the 2-dimensional spheres $S^{2}$. 
Proof. By Lemma $12, \mathcal{N}\left(C_{n}(s, t)\right)$ collapses to a 2-dimensional subcomplex $\Delta$, whose maximal simplices $M(\Delta)=\left\{\tau_{k}^{i} \mid i \in\{1,2\}, k \in[n]\right\}$, where $\tau_{k}^{1}=\{s+k, t+k, n-t+k\}$ and $\tau_{k}^{2}=\{n-s+k, t+k, n-t+k\}$. For any integer $i$, let $\Gamma_{i}:=\{t+i(\bmod n), n-t+$ $i(\bmod n), s+i(\bmod n), s+2 t+i(\bmod n)\}$ be a 3 -simplex. Clearly, the simplicial complexes $\tau_{k}^{1} \cup \tau_{s+t+k}^{2} \cup \tau_{2 t+k}^{1} \cup \tau_{n-t+s+k}^{2}=B d\left(\Gamma_{k}\right)$ and $\tau_{n-s-t+k}^{1} \cup \tau_{k}^{2} \cup \tau_{n-s+t+k}^{1} \cup \tau_{2 t+k}^{2}=B d\left(\Gamma_{n-s-t+k}\right)$. Hence, each 1-simplex of $\Delta$ is part of $\operatorname{Bd}\left(\Gamma_{i}\right)$ for some $i \in[n]$. Thus $\Delta=\bigcup_{1 \leqslant i \leqslant n} B d\left(\Gamma_{i}\right)$.

In the rest of the proof, if we write an integer $x$ as a vertex of $\Gamma_{i}$ for some $i$, then it is understood that we are taking $x(\bmod n)$. Now $B d\left(\Gamma_{n-s-t+k}\right) \cap B d\left(\Gamma_{k}\right)=\{t+k, n-t+k\}$ and $B d\left(\Gamma_{k}\right) \cap B d\left(\Gamma_{k+s+t}\right)=\{s+k, s+2 t+k\}$, i.e., $B d\left(\Gamma_{k}\right)$ shares a common 1-simplex $\{t+k, n-t+k\}$ with $B d\left(\Gamma_{n-s-t+k}\right)$ and a common 1-simplex $\{s+k, s+2 t+k\}$ with $\Gamma_{s+t+k}$.

To show that each component of $\Delta$ is homotopy equivalent to a garland of the 2dimensional spheres, it is enough to show that each vertex $k$ belongs to exactly the boundaries of two 3 -simplices. Let $x \in[n]$. There exists $k \in[n]$ such that $x \in B d\left(\Gamma_{k}\right)$, i.e., $x \in\{t+k, n-t+k, s+k, s+2 t+k\}$. It is clear from the above discussion that there exists $k^{\prime} \neq k$ such that $x \in B d\left(\Gamma_{k^{\prime}}\right)$, i.e., $x \in\left\{t+k^{\prime}, n-t+k^{\prime}, s+k^{\prime}, s+2 t+k^{\prime}\right\}$. Since $n=4 t, \Gamma_{k+2 t}=\Gamma_{k} \forall k \in[n]$. Let $x=t+k$. If $x \equiv t+k^{\prime}$, then $k \equiv k^{\prime}$, a contradiction. If $x \equiv n-t+k^{\prime}$, then $k^{\prime} \equiv k-2 t$. But $\Gamma_{k-2 t}=\Gamma_{k}$. Since, $t+k \equiv s+k^{\prime}$ implies that $k^{\prime} \equiv t-s+k$ and $t+k \equiv s+2 t+k^{\prime}$ implies that $k^{\prime} \equiv k-t-s$, we conclude that $x \in B d\left(\Gamma_{k}\right)$ and $B d\left(\Gamma_{n-s-t+k}\right)$ only. By a similar argument as the one above, we can easily verify that, $n-t+k \in B d\left(\Gamma_{k}\right) \cap B d\left(\Gamma_{n-s-t+k}\right), s+k \in B d\left(\Gamma_{k}\right) \cap B d\left(\Gamma_{s+t+k}\right)$ and $s+2 t+k \in B d\left(\Gamma_{k}\right) \cap B d\left(\Gamma_{s+t+k}\right)$ only.

Thus $x$ belongs to either $B d\left(\Gamma_{k}\right) \cap B d\left(\Gamma_{n-s-t+k}\right)$ or $B d\left(\Gamma_{k}\right) \cap B d\left(\Gamma_{s+t+k}\right)$ only.

A $d$-dimensional pseudo manifold is a pure $d$-dimensional simplicial complex such that every $(d-1)$-simplex is a face of exactly two $d$-simplices. A (topological) $n$-manifold is a hausdorff space $X$ such that every point $x \in X$, has a neighborhood which is homeomorphic to $\mathbb{R}^{n}$. A 2-manifold is called a surface.

Theorem 14. Let $s, t \in\left\{1,2, \ldots,\left\lfloor\frac{n}{2}\right\rfloor\right\}$ such that $2 s, 2 t, 2(s+t), 3 s+t, 3 t+s, 3 s-t, 3 t-$ $s, 4 s, 4 t \neq n, 3 s \neq t$ and $3 t \neq s$. Then each connected component of $\mathcal{N}\left(C_{n}(s, t)\right)$ is homotopy equivalent to connected sum of tori.

We recall the following result to prove Theorem 14 .

Proposition 15. (Theorem 3A.3, [5])

If $C$ is a chain complex of free abelian groups, then there exist short exact sequences

$$
0 \longrightarrow H_{n}(C ; \mathbb{Z}) \otimes \mathbb{Z}_{2} \longrightarrow H_{n}\left(C ; \mathbb{Z}_{2}\right) \longrightarrow \operatorname{Tor}\left(H_{n-1}(C ; \mathbb{Z}), \mathbb{Z}_{2}\right) \longrightarrow 0
$$

for all $n$ and these sequences split.

Proof of Theorem 14. By Lemma 12, $\mathcal{N}\left(C_{n}(s, t)\right)$ collapses to a subcomplex $X$, with $M(X)=\left\{\tau_{k}^{i} \mid i \in\{1,2\}, k \in[n]\right\}$, where $\tau_{k}^{1}=\{s+k, t+k, n-t+k\}$ and $\tau_{k}^{2}=$ $\{n-s+k, t+k, n-t+k\}$. 


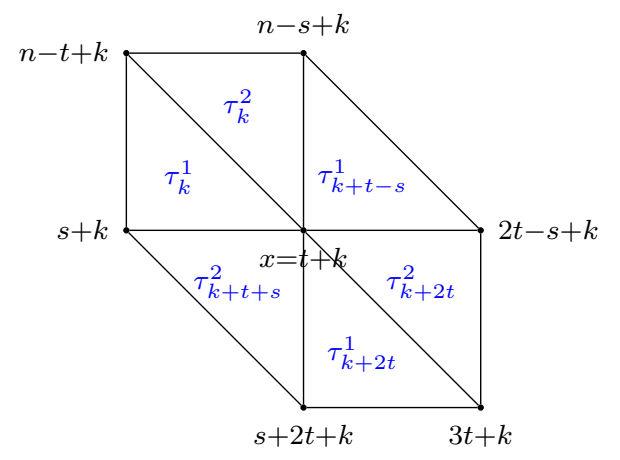

(a)

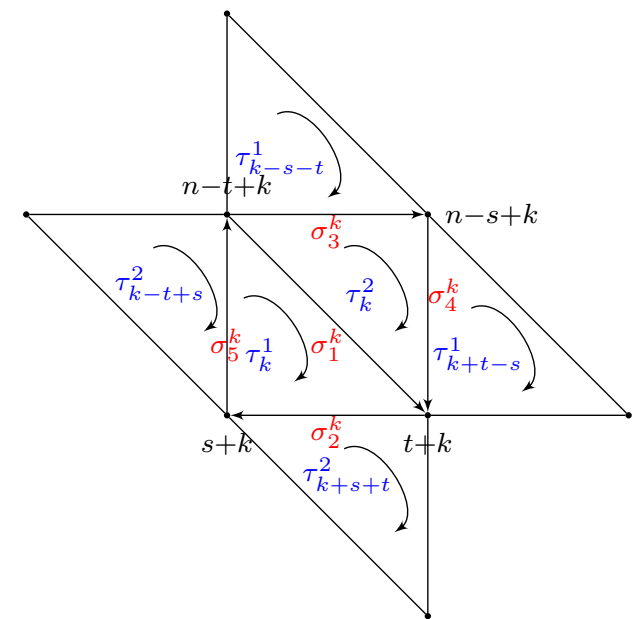

(b)

Figure 2

Claim 16. $X$ is a 2-dimensional pseudo manifold.

Proof of Claim 16. It is enough to show that any 1-dimensional face of $\tau_{k}^{1}$ and $\tau_{k}^{2}$, i.e., $\sigma_{1}^{k}=\{t+k, n-t+k\}, \sigma_{2}^{k}=\{s+k, t+k\}, \sigma_{3}^{k}=\{n-t+k, n-s+k\}, \sigma_{4}^{k}=\{t+k, n-s+k\}$ and $\sigma_{5}^{k}=\{s+k, n-t+k\}$ are faces of exactly two 2-dimensional simplices of $X$.

Clearly, $\sigma_{1}^{k}$ is a face of $\tau_{k}^{1}$ and $\tau_{k}^{2}$. By Lemma $12,\left(\sigma_{1}^{k}, N(k)\right)$ is a collapsible pair and therefore $\sigma_{1}^{k}$ is not a face of any $\tau_{k^{\prime}}^{1}$ or $\tau_{k^{\prime}}^{2}$ for all $k^{\prime} \neq k$.

$\sigma_{2}^{k} \subset \tau_{k+s+t}^{2}=\{k+t, s+2 t+k, s+k\}$. Since $s, t<\frac{n}{2}, s+2 t+k \equiv n-t+k$ implies that $3 t+s=n$, which is not possible. Hence $\tau_{k+s+t}^{2} \neq \tau_{k}^{1}$. Further, $3 s-t$ and $3 t-s \neq n$ implies that $N(s+k) \cap N(t+k)=\{k, s+t+k\}$. Since, $\sigma_{2}^{k} \nsubseteq \tau_{s+t+k}^{1}, \sigma_{2}^{k}$ is a face of $\tau_{k}^{1}$ and $\tau_{k+s+t}^{2}$ only. Since $n-t+k \equiv s+(k-s-t)$ and $n-s+k \equiv t+(k-s-t)$, we see that, $\sigma_{3}^{k}$ is a face of $\tau_{k-s-t}^{1}$ and $\tau_{k}^{2}$ only.

$\sigma_{4}^{k} \subset \tau_{k+t-s}^{1}=\{t+k, 2 t-s+k, n-s+k\}$ and $\sigma_{4}^{k} \nsubseteq \tau_{k+t-s}^{2}$. Since $2 t-s+k \equiv n-t+k$ implies that $3 t-s=n$, we see that $\tau_{k+t-s}^{1} \neq \tau_{k}^{2}$. Further, $2(s+t), 3 s+t, 3 t+s \neq n$ implies that $N(t+k) \cap N(n-s+k)=\{k, k-t+s\}$. Thus $\sigma_{4}^{k}$ is a face of $\tau_{k}^{2}$ and $\tau_{k+t-s}^{1}$ only. Since $s+k=t+(k-t+s)$ and $n-t+k=n-s+(k-t+s)$, we see that, $\sigma_{5}^{k}$ is a face of $\tau_{k-t+s}^{2}$ and $\tau_{k}^{1}$ only.

Claim 17. $\|X\|$, the geometric realization of $X$, is a surface, i.e., a 2-manifold.

Proof of Claim 17. Let $x \in\|X\|$. Since, $X$ is a pseudomanifold, if $x$ belongs to interior of some 1-simplex or 2-simplex, then we can easily construct an open neighborhood of $x$, homeomorphic to $\mathbb{R}^{2}$. Assume $x$ is a 0 -simplex of $X$. Without loss of generality, we can assume that $x=t+k$, for some $k \in[n]$. Then $x \in \tau_{k}^{1}, \tau_{k}^{2}, \tau_{k+t-s}^{2}, \tau_{k+2 t}^{2}, \tau_{k+2 t}^{1}$ and $\tau_{k+t+s}^{2}$ (see Figure 2a). Using the fact that $2(s+t), 3 t-s, 3 t+s, 4 t \not \equiv 0$, it can be easily checked that $|\{s+k, n-t+k, n-s+k, 2 t-s+k, 3 t+k, s+2 t+k\}|=6$. Since 
$N(x)=\{k, k+t-s, k+2 t, k+s+t\}, x \notin \tau_{m}^{1}$ or $\tau_{m}^{2}$ for all $m \neq k, k+t-s, k+2 t, k+s+t$. Hence we can easily construct a neighborhood of $x$ in $\|X\|$, which is homeomorphic to $\mathbb{R}^{2}$. Thus $X$ is a surface.

We now define the orientations for simplices of $X$. For any oriented simplex $\sigma$ we write $+\sigma$, if it is positively oriented and $-\sigma$, otherwise. We denote any positively oriented 2simplex with vertex set $\{a, b, c\}$ by $\langle a, b, c\rangle$ and 1 -simplex with vertex set $\{a, b\}$ by $\langle a, b\rangle$.

For any $k \in[n]$, we define $+\tau_{k}^{1}=\langle s+k, n-t+k, t+k\rangle$ and $+\tau_{k}^{2}=\langle n-t+k, n-$ $s+k, t+k\rangle$. Further, we define $+\sigma_{1}^{k}=\langle n-t+k, t+k\rangle,+\sigma_{2}^{k}=\langle t+k, s+k\rangle,+\sigma_{3}^{k}=$ $\langle n-t+k, n-s+k\rangle,+\sigma_{4}^{k}=\langle n-s+k, t+k\rangle$ and $+\sigma_{5}^{k}=\langle s+k, n-t+k\rangle$. Observe that any 1 -simplex of $X$ is equal to $\sigma_{i}^{k}$ for some $k \in[n]$ and $i \in\{1,2,3,4,5\}$.

Let $C=\left(C_{i}, \partial_{i}\right)$ be the simplicial chain complex of $X$ with coefficients in $\mathbb{Z}_{2}$. Since, there are $n 0$-simplices in $X, C_{0} \cong \mathbb{Z}_{2}^{n}$. Further, since there are $2 n$ simplices of dimension 2 and $X$ is a pseudo manifold, $C_{1} \cong \mathbb{Z}_{2}^{3 n}$ and $C_{2} \cong \mathbb{Z}_{2}^{2 n}$. Since there is no simplex of any other dimension in $X, C_{i}=0$ for all $i \neq 1,2,3$. Thus

$$
C=0 \longrightarrow \mathbb{Z}_{2}^{2 n} \stackrel{\partial_{2}}{\longrightarrow} \mathbb{Z}_{2}^{3 n} \stackrel{\partial_{1}}{\longrightarrow} \mathbb{Z}_{2}^{n} \stackrel{\partial_{0}}{\longrightarrow} 0
$$

Let $p$ be the number of connected components of $X$. It is well known that $H_{0}\left(X ; \mathbb{Z}_{2}\right) \cong \mathbb{Z}_{2}^{p}$. Since $p \geqslant 1, \operatorname{Rank}\left(\partial_{1}\right) \leqslant n-1$. If Ker $\partial_{1} \cong \mathbb{Z}_{2}^{r}$, then $r \geqslant 3 n-n+1=2 n+1$. Since $\operatorname{Rank}\left(\partial_{2}\right)$ can be at most $2 n, H_{1}\left(X ; \mathbb{Z}_{2}\right) \neq 0$. From Proposition $15, H_{1}\left(X ; \mathbb{Z}_{2}\right) \cong H_{1}(X ; \mathbb{Z}) \otimes \mathbb{Z}_{2}$ $\oplus \operatorname{Tor}\left(H_{0}(X ; \mathbb{Z}), \mathbb{Z}_{2}\right)$. Since $H_{0}(X ; \mathbb{Z}) \cong \mathbb{Z}^{p}$, $\operatorname{Tor}\left(H_{0}(X) ; \mathbb{Z}_{2}\right)=0$. So $H_{1}(X ; \mathbb{Z})=0$, implies that $H_{1}\left(X ; \mathbb{Z}_{2}\right)=0$, which is a contradiction. Hence $H_{1}(X ; \mathbb{Z}) \neq 0$.

Let $D=\left(D_{i}, d_{i}\right)$ be the simplicial chain complex of $X$ with $\mathbb{Z}$ coefficients. Then

$$
D=0 \longrightarrow \mathbb{Z}^{2 n} \stackrel{d_{2}}{\longrightarrow} \mathbb{Z}^{3 n} \stackrel{d_{1}}{\longrightarrow} \mathbb{Z}^{n} \stackrel{d_{0}}{\longrightarrow} 0
$$

Let $c=\sum_{k \in[n]}\left(\left(+\tau_{k}^{1}\right)+\left(+\tau_{k}^{2}\right)\right)$ be a 2-chain. It can be easily verified (see Figure $2 \mathrm{~b}$ ) that $\sigma_{1}^{k}$ has +ve orientation in $\tau_{k}^{1}$ and -ve orientation in $\tau_{k}^{2} . \sigma_{2}^{k}$ has +ve orientation in $\tau_{k}^{1}$ and - ve in $\tau_{k+s+t}^{2}$. Similarly $\sigma_{3}^{k}$ has + ve orientation in $\tau_{k}^{2}$ and - ve in $\tau_{k-t-s}^{1}$. The simplex $\sigma_{4}^{k}$ having +ve orientation in $\tau_{k}^{2}$ and - ve in $\tau_{k+t-s}^{1}$, and $\sigma_{5}^{k}$ has +ve orientation in $\tau_{k}^{1}$ and -ve in $\tau_{k-t+s}^{2}$. Since $X$ is a pseudo manifold, each 1-simplex of $X$ will occur twice in $d_{2}(c)$, once with + ve sign and once with - ve sign. Hence $d_{2}(c)=0$. Since $\tau_{k}^{i} \neq 0$ in $D_{2}=\mathbb{Z}^{2 n}, 0 \neq c \in$ Ker $d_{2}$. Hence $H_{2}(X ; \mathbb{Z}) \neq 0$.

From Claim 17, each component of $X$ is a compact surface. From the classification of surfaces, we know that any connected compact surface is homeomorphic either to $S^{2}$, to a connected sum of tori or to a connected sum of projective planes.

Since $H_{1}(X ; \mathbb{Z}) \neq 0$, the connected components of $X$ cannot be homeomorphic to $S^{2}$. Further, since $H_{2}(S ; \mathbb{Z})=0$ for any non orientable surface $S$, the connected components of $X$ cannot be homeomorphic to a connected sum of projective planes. Hence each connected component of $X$ is homeomorphic to a connected sum of tori.

Let $\Delta$ be a simplicial complex. An $m$-path in $\Delta$ is a sequence $\sigma_{1} \ldots \sigma_{t}, t \geqslant 2$ of $m$ simplices such that $\sigma_{i}$ and $\sigma_{i+1}$ have a common $(m-1)$-dimensional face, for all $1 \leqslant i \leqslant$ 

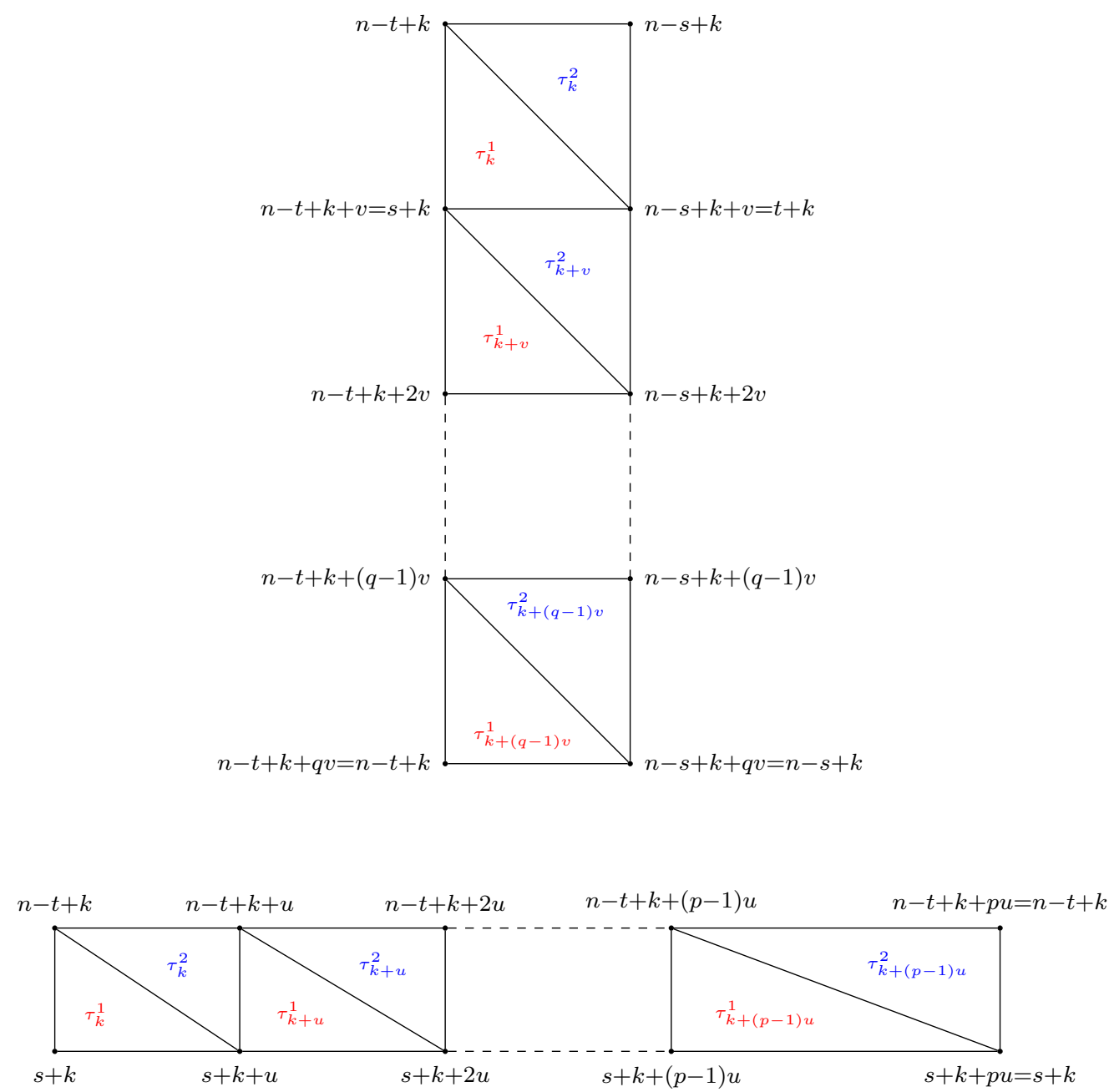

Figure 3

$t-1$. Further, if $\sigma_{1}$ and $\sigma_{t}$ have a common $(m-1)$-dimensional face, then it is said to be closed $m$-path.

Proof of Theorem \%. Using Lemma $12, \mathcal{N}\left(C_{n}(s, t)\right)$ collapses to a subcomplex $X$ with $M(X)=\left\{\tau_{k}^{i} \mid i \in\{1,2\}, k \in[n]\right\}$, where $\tau_{k}^{1}=\{s+k, t+k, n-t+k\}$ and $\tau_{k}^{2}=$ $\{n-s+k, t+k, n-t+k\}$. Let $u=t-s$ and $v=t+s$. Let $k \in[n]$. Since, $s+u+k=t+k$ and $n-t+u+k=n-s+k$, we see that $\tau_{k}^{2}$ and $\tau_{k+u}^{1}$ have a common 1-simplex $\{n-s+k, t+k\}$. Further, $n-s+k+v=t+k$ and $n-t+k+v=s+k$ implies that $\tau_{k}^{1}$ and $\tau_{k+v}^{2}$ have a common 1-simplex $\{s+k, t+k$,$\} . Since \tau_{k}^{1}$ and $\tau_{k}^{2}$ have a common 1-simplex $\{t+k, n-t+k\}$, we conclude that $\tau_{k}^{1} \tau_{k}^{2}, \tau_{k}^{2} \tau_{k+u}^{1}$ and $\tau_{k}^{1} \tau_{k+v}^{2}$ are 2-paths in $X$.

(i) $s=\frac{p-q}{2}$ and $t=\frac{p+q}{2}$. 
In this case $q=t-s=u$ and $p=t+s=v$. Now, $\omega=\tau_{k}^{1} \tau_{k}^{2} \tau_{k+u}^{1} \tau_{k+u}^{2} \ldots$ $\tau_{k+(p-1) u}^{1} \tau_{k+(p-1) u}^{2}$ and $\Gamma=\tau_{k}^{2} \tau_{k}^{1} \tau_{k+v}^{2} \tau_{k+v}^{1} \ldots \tau_{k+(q-1) v}^{2} \tau_{k+(q-1) v}^{1}$ are 2-paths in $X$. Since $k+p u$ and $k+q v \equiv k(\bmod \mathrm{n}), \omega$ (see the horizontal rectangular strip of Figure 3 ) and $\Gamma$ (see the vertical rectangular strip of Figure 3 ) are closed paths in $X$. Thus the simplices of $X$ can be arranged in a rectangular grid of order $(q+1) \times(p+1)$ as depicted in Figure 4 . Since $X$ has $2 n$ 1-dimensional simplices, to prove that this rectangular grid gives a triangulation of a torus, it is enough to show that there is no identification among the vertices other than that shown in Figure 4. Any vertex of $C_{n}(s, t)$ can be written as $n-t+k$ for some $k \in[n]$ and therefore can be made as the left uppermost corner vertex $a_{1,1}$ of this grid. Hence, it is enough to show that $n-t+k=a_{1,1} \neq a_{i, j}$ unless $i \in\{1, q+1\}$ and $j \in\{1, p+1\}$, i.e., $n-t+k$ lies only on the four corners of the grid. But, since $a_{i, j}=(i-1) p+n-t+k+(j-1) q$, $a_{i, j}=n-t+k$ implies that $(i-1) p+(j-1) q \equiv 0(\bmod \mathrm{n})$. Further, since $\operatorname{gcd}(p, q)=1$, this is only possible if $i \in\{1, q+1\}$ and $j \in\{1, p+1\}$.

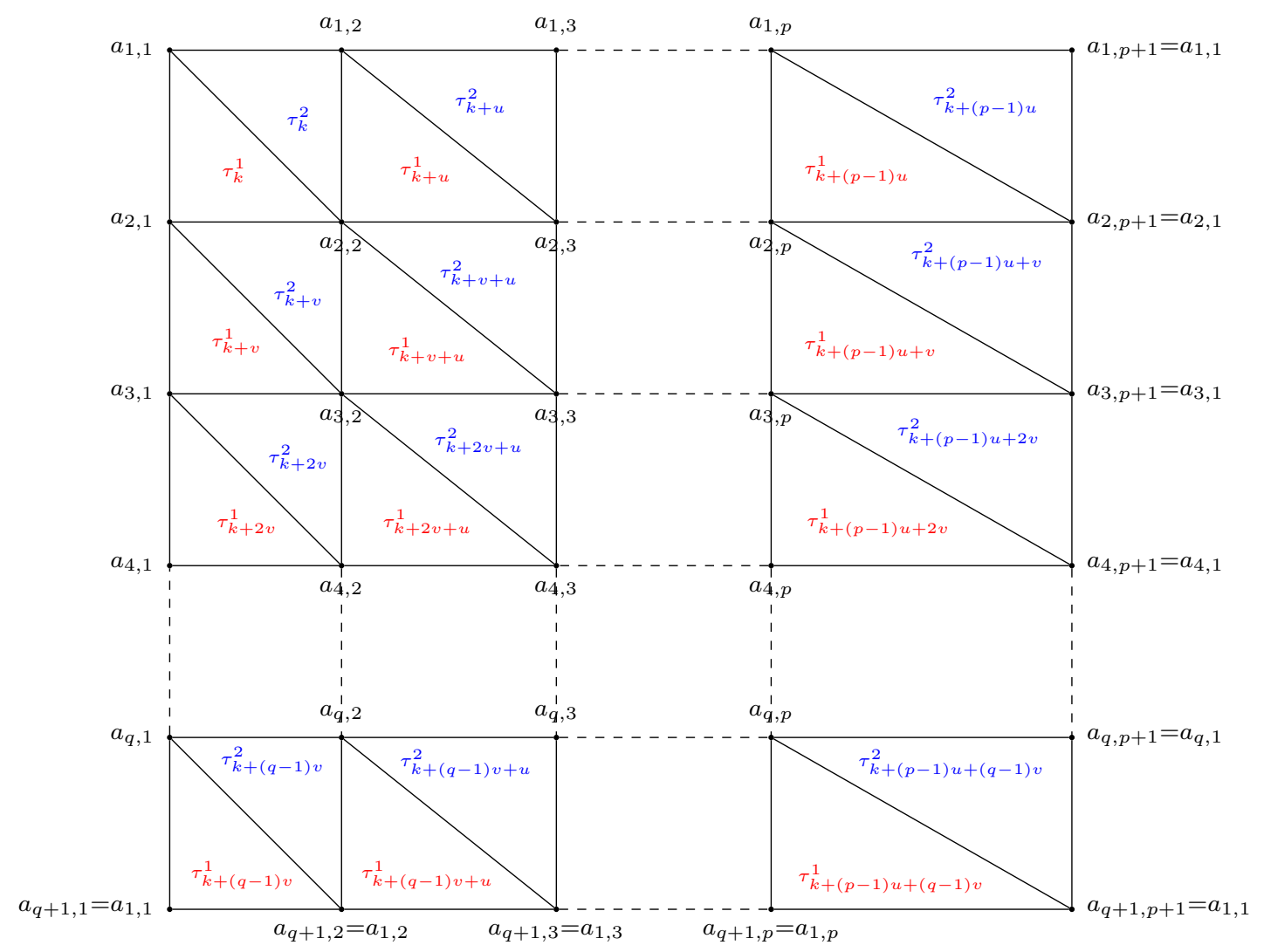

Figure 4: $u=t-s, v=t+s, a_{i, j}=(i-1) v+n-t+k+(j-1) u, 1 \leqslant i \leqslant q+1,1 \leqslant j \leqslant p+1$.

(ii) $s=\frac{p^{2}-q}{2}$ and $t=\frac{p^{2}+q}{2}$.

In this case, $v=s+t=p^{2}$ and $u=t-s=q$. Since $p q=n$, we have the closed 2-paths $\tau_{k}^{1} \tau_{k}^{2} \tau_{k+u}^{1} \tau_{k+u}^{2} \ldots \tau_{k+(p-1) u}^{1} \tau_{k+(p-1) u}^{2}$ and $\tau_{k}^{2} \tau_{k}^{1} \tau_{k+v}^{2} \tau_{k+v}^{1} \ldots \tau_{k+(q-1) v}^{1} \tau_{k+(q-1) v}^{1}$. 
In this case also, we can arrange the simplices of $X$ in a rectangular grid of order $(q+1) \times(p+1)$, as depicted in Figure 4. By an argument similar as of the case $(i)$, to prove that this rectangular grid gives a triangulation of a torus, it is enough to show that $n-t+k=a_{1,1} \neq a_{i, j}$ unless $i \in\{1, q+1\}$ and $j \in\{1, p+1\}$. But, since $a_{i, j}=(i-1) v+n-t+k+(j-1) u, a_{i, j}=n-t+k$ implies that $(i-1) v+(j-1) u \equiv 0(\bmod \mathrm{n})$. Since $v=p^{2}, u=q,(i-1) p^{2}+(j-1) q \equiv 0(\bmod \mathrm{n})$.

This is only possible when $i \in\{1, q+1\}$ and $j \in\{1, p+1\}$ as $\operatorname{gcd}(p, q)=1$.

\section{Acknowledgements}

The author would like to thank anonymous referees for their helpful suggestions which led to a significant improvement in the presentation of this article.

\section{References}

[1] E. Babson and D. N. Kozlov, Complexes of graph homomorphisms. Israel J. Math, 152: 285-312, 2006.

[2] E. Babson and D. N. Kozlov, Proof of the Lovász conjecture, Annals of Math. (2), 165: 965-1007, 2007.

[3] A. Björner and M. de Longueville, Neighborhood complexes of stable Kneser graphs, Combinatorica, 23(1): 23-34, 2003.

[4] Anders Björner, Topological methods. Handbook of combinatorics, Elsevier, Amsterdam 1,2: 1819-1872, 1995.

[5] A. Hatcher, Algebraic topology, Cambridge University Press, 2002.

[6] M. Kneser, Aufgabe 360, Jahresbericht der Deutschen Mathematiker-Vereinigung, 58, 1955.

[7] D. N. Kozlov, Combinatorial algebraic topology, Springer Verlag, Berlin, 2008.

[8] L. Lovász, Kneser's conjecture, chromatic number and homotopy, J. Combin. Theory Ser. A, 25: 319-324, 1978.

[9] J. R. Munkres, Topology, Second edition, Prentice Hall, Upper saddle River, 2000.

[10] N. Nilakantan and S. Shukla, Neighborhood complexes of some exponential graphs, Electron. J. Combin. 23(2): \#P2.26, 2016. 